profession of sanitary engineering differs a good deal from country to country, and there is no general agreement on what the basic training of a sanitary engineer should be. Generally, however, this training falls somewhere between the two extremes represented by United States and British practice. In the former the student is trained in a university school of sanitary engineering, whereas in Great Britain the sanitary engineer is a man who is first trained in civil engineering and then specializes in the field of sanitation or public health. It may be mentioned, however, that in addition to a sanitary engineer thus defined, there are in Great Britain many men working in the field of sanitation whose basic training was in chemistry or biology and who are usually members of the Koyal Sanitary Institute or the Institute of Sewage Purification, or who are professional associates of the Institution of Water Engineers. In this matter of training, there is probably no one best way, and the method found most efficient for any particular country will depend on the complexity and importance of the problems to be solved.

There was general agreement at the seminar that the ultimate formation of an international association of sanitary engineers is desirable. Here it may be recalled that a somewhat similar body-the International Water Supply Association, formed in 1947is flourishing strongly and is holding its second congress in Paris this year. Some of the problems which would be encountered in introducing any standardization in sanitary matters in Europe were apparent in the discussion of a paper by Prof. U. A. Corti, of Switzerland, on the possible development of international standards for drinking water. Until recently there was general agreement on the more obvious standards required in a potable water - the absence, for example, of toxic metals and of pathogenic bacteria. It is clear, however, that if an attempt were made at present to lay down precise standards for all the minor constituents found in natural waters, or added during the course of treatment, a wide difference of opinion would be revealed. This is obvious, indeed, from the discussions which have followed recent proposals to add fluoride to water to reduce the incidence of dental decay. In any proposal to develop international standards the first step would seem to be to introduce standard methods of chemical analysis and bacteriological examination, and this point was made by several speakers during the discussion.

An important part of the Seminar was given up to discussions on the treatment and disposal of sewage, particularly from single houses and small communities. Installations of this kind, consisting usually of a septic tank and percolating filter, are in common use in some parts of the Continent and, to a small extent, in rural districts in Great Britain. Although they are one of the earliest types of treatment plant, surprisingly little is known about the principles on which their operation depends. This applies also to the disposal of the final effluent - which may contain pathogenic organisms-by subsurface irrigation, and much more work on this subject is required. It may be mentioned, for example, that in some parts of Great Britain lack of knowledge of the movement of liquids underground, and of the removal of bacteria from them in passing through various formations, limits the disposal of household refuse by controlled tipping in abandoned gravel pits-a method which has the advantage of being the cheapest form of disposal and of reclaiming otherwise derelict land.
The seminar concluded with a very well-documented paper by Prof. M. Petrick, of Yugoslavia, on the utilization of night-soil and sewage sludge in agriculture, a subject on which there is now a considerable amount of quantitative information. There was general agreement that the series of seminars for European sanitary engineers should be continued, and it has since been announced that the third will be held in Great Britain in October this year.

\section{BIOLOGICAL ASSESSMENT OF CHEMOTHERAPEUTICS}

$T$

HE Biological Methods Group of the Society of Public Analysts and Other Analytical Chemists held its summer meeting in Nottingham during June 12-13, at the invitation of the directors of Boots Pure Drug Co., Ltd.

On June 12, the members of the Group visited the Pharmacology and Bacteriology Divisions of the Research Department, and the Bio-Assay Division of the Standards Department. In the Pharmacology Division demonstrations were given of the methods used for examining new substances for chemotherapeutic activity against the organisms of some tropical diseases, including amœbiasis, leishmaniasis, schistosomiasis and trypanosomiasis. Particularly interesting was the exhibit showing all stages of the life-cycle of Schistosoma mansoni, including ova, miracidia, cercariæ and adult worms. A demonstration was given of the value of the phase-contrast microscope for the examination of living, unstained Protozoa.

A cartesian diver apparatus was shown in use to measure the rates of oxygen uptake of small numbers of Trypanosoma congolense under various conditions. The apparatus was based on that first described by Linderstrøm-Lang: it has been adapted for use at $37^{\circ} \mathrm{C}$., and to allow addition of substrates and inhibitors to the respiring organisms.

The examination of compounds for anti-tumour activity was demonstrated. Transplanted tumours such as the Walker carcinoma in rats and the mouse sarcoma 37 were shown, and the effect of the administration of such substances as nitrogen mustard and triethylene melamine on their development.

Methods of assessment of pharmacodynamic activity in new compounds which were shown included the estimation of local anæsthetic activity by a modification of the Bulbring-Wajda method, and the estimation of antihistamine and bronchodilator activities by the inhibition of bronchoconstriction produced in guinea pigs by the intravenous injection of histamine and other bronchoconstrictor agents. A demonstration was given of a possible method of assessing cortisone-like activity by the inhibition of granuloma-formation around implanted cotton-wool pellets and other foreign bodies in the rat.

Methods for examining acute and chronic systemic toxicity were demonstrated: a simple device was shown for dispensing drug-containing diet to mice in such a way that the daily consumption can be accurately measured. Several methods of assessing local toxicity and tissue damage were shown, including intradermal injection in guinea pigs, prolonged surface application to the shaved abdomen of mice under a fluid-tight cap, and the use of a small glass vacuum- 
cup to extract histamine-containing tissue fluid through the skin of a rat immediately over the site of a subcutaneous injection of an irritant substance.

In the Bacteriology Division, demonstrations were given of the various techniques used in the screening of new substances for possible activity against bacteria, viruses, and pathogenic fungi. In the Tuberculosis Section, the animal room was shown in which infected animals are maintained behind glass in a continuous current of air drawn into the room and expelled through filters. Laboratory demonstrations showed in vitro tests by the floating pellicle technique, and in vivo tests using guinea pigs and intracerebrally infected mice, and the method of assessment of drug activity on the basis of gross pathology. Groups of lungs taken from tuberculous mice, treated with known drugs such as $p$-aminosalicylic acid, thiacetazone, streptomycin, isonicotinic acid hydrazide, etc., were shown.

In the Virus Section, the use of bacteriophage as a screening virus was demonstrated. Methods with embryonated eggs for screening against influenza $A$ and other viruses were shown, and with chicks against the Rous sarcoma virus. In the General Chemotherapy Section, a demonstration was given of an in vivo test in mice for the assessment of delay activity in penicillin preparations.

The Antibiotic Section showed the types of smallscale fermenters used for the experimental study of antibiotic production by new mutants of Penicillium chrysogenum or by newly isolated organisms. These included revolving tubes, shaker flasks, and larger fermenters. Methods of isolation and study of new organisms were displayed, as also were the biochemical studies carried out on the cultures during fermentations.

In the Bio-Assay Division, demonstrations included the assay of routine batches of insulin using the mouse convulsion method, and the determination of the delay activity exhibited by protamine zine insulin in rabbits. The use of thermocouples for the determination of the rectal temperature of rabbits in pyrogen tests was shown. Other exhibits included the sulphated ox blood method of heparin assay, the rat 'line test' technique for the estimation of vitamin $D$ and the standardization of posterior pituitary extract using the isolated uterus of the guinea pig.

On June 13 the Group visited the Veterinary Science Division at Thurgarton Priory. Demonstrations were given of the symptomatology and pathology of blackhead infection in turkeys, and methods of producing infection artificially were discussed: the use of artificially infected poults for drug assessment was described. Methods employed for the evaluation of new drugs against the common helminth parasites of domestic animals included a simple in vivo screening method using the free-living nematode Turbatrix aceti, cultured in serial dilutions of the drug.

A demonstration of work on Johnes disease in cattle included pathological specimens showing massive infection of the tissues. A brief account of the naturally occurring disease was given, and attempts at treatment with streptomycin and thiacetazone were described. A biochemical study of the digestive metabolism of ruminants was discussed. The changes which occur in the leucocyte picture and in certain blood constituents of the ewe in late pregnancy were described, and their possible relation to the adrenocortical hormones was discussed. An account was given of a method of field evaluation of sheep dips, in which an attractant solution is applied to the skin and wool of sheep at intervals after dipping, and the efficiency determined by the effect on blowflies attempting to oviposit on the wool.

Demonstrations were given of the methods used in the evaluation of disinfectants for dairy use. Disinfectants for hatching eggs were tested on eggs infected on the surface with a Salmonella culture. Disinfectants for dairy utensils were assessed by their effect on plate counts on standard metal strips infected with cultures suspended in milk. Field trials of dairy disinfectants under working conditions were described.

The meeting ended with a short scientific session at which papers by Dr. G. F. Somers on "The Assay of Adrenaline in Crearns", and by Dr. P. W. Muggleton on "The Standardization of Pertussis Vaccine" were read and discussed. The chairman of the Group, Dr. H. O. J. Collier, expressed the appreciation of the members to Mr. W. A. Broom and his colleagues for organizing the meeting.

\section{TRANSPARENT CONDUCTING FILMS OF GOLD}

$\mathrm{N}$ an article in Nature describing recent work in 1 the Light Division of the National Physical Laboratory, Teddington, on methods of producing transparent electrically conducting films on glass ${ }^{1}$, the films then mentioned were oxide films; and, although the conductivity of some, especially tin oxide, had been abnormally enhanced by special treatment, the modus operandi is not yet fully understood. One object of the work was to provide means for heating the glass electrically; but it was noted that the resistance of the films was rather high, making necessary the use of voltages of some hundreds for the effective de-icing of coated aircraft windows in adverse conditions.

A further investigation has rapidly followed this, by the same workers, and with the same practical aim. The results are noteworthy because they touch upon a rather different field of research. It has long been known that metallic films, made by no matter what process, almost always have a much greater resistivity than the bulk metal, even after allowance for an expected increase due to the thinness of the films. This discrepancy between observed and predicted resistivities is assignable to the difference between the granular or non-coherent structure of the actual films, and the homogeneous plane-parallel model assumed in the theory.

The starting-point of the new investigation was a study of the influence which the condition-and material of the substrate might have upon the properties of superposed gold films. For example, a gold film laid down on glass cleaned with dry precipitated chalk was found to have a resistivity less than half that of similar films on glass cleaned by more ordinary methods, while the colour of the film was a dull yellowish-green instead of the familiar bluish-green. These experiments were made with gold films about $70 \mathrm{~A}$. in thickness, laid down under strictly controlled conditions by cathodic sputtering. Similar films were then laid down on glass freshly coated with substrate films of various oxides, also made by sputtering. In some such cases, especially with substrates of the oxides of cadmium, lead, bismuth and zinc, the properties of the gold film were even 